\title{
Prevalence of cognitive impairment in acute ischaemic stroke and use of Alberta Stroke Programme Early CT Score (ASPECTS) for early prediction of post-stroke cognitive impairment
}

\author{
Ahmed Esmael, Mohammed Elsherief, Khaled Eltoukhy \\ Neurology Department, Faculty of Medicine, Mansoura University, Dakahlia, Egypt
}

\begin{abstract}
Aim of the study. This study aims to assess the prevalence of post-stroke cognitive impairment, and to evaluate the correlation of ASPECTS with impaired cognition.

Materials and methods. 150 patients presenting with acute middle cerebral artery territory ischaemic stroke were included in this study. Risk factors of ischaemic stroke and the initial NIHSS were determined. An initial and a follow-up non-contrast CT brain were carried out after seven days which were assessed by ASPECTS. The prevalence of cognitive impairment was determined by MoCA during the follow up of patients after three months. Correlations of ASPECTS, NIHSS and MoCA were done by Spearman correlation. Multivariate logistic regression analysis was carried out for the independent variables of cognitive impairment.

Results. The prevalence of post-stroke cognitive impairment in this study, according to the threshold for cognitive impairment with a MoCA score of 25 or less, was $25.3 \%$ (38 patients). Significant positive correlations between ASPECTS and total MoCA test domains were found $(r=0.73$ and $p=0.002)$. Logistic regression analysis demonstrated that the independent factors associated with cognitive impairment were older age, certain domains of the MoCA test like executive functions, memory, attention, language, NIHSS, HTN, and ASPECTS.
\end{abstract}

Conclusions and clinical implications. There is a prevalence of cognitive impairment in about $25 \%$ of patients after three months of follow-up in cases with acute ischaemic stroke. ASPECTS is directly correlated with cognitive impairment, and may be considered as a biomarker of post-stroke cognitive impairment.

Key words: stroke, Alberta Stroke Programme Early CT Score, National Institutes of Health Stroke Scale, cognitive impairment (Neurol Neurochir Pol 2021; 55 (2): 179-185)

\section{Introduction}

Stroke is the third leading cause of death, and is considered to be an important cause of disability and impaired cognition $[1,2]$. Post-stroke cognitive impairment is a common but neglected sequel compared to other neurological deficits [3]. Different domains of cognition, such as attention, concentration, memory, language, and executive functions may be affected in stroke survivors [4].

Up to $50 \%$ of all cases with ischaemic stroke show diminished or below-average performance of cognitive functions. Impaired processing speed, attention and working memory are frequently affected [5]. Also, a high proportion of stroke survivors have cognitive impairment within three months after stroke, as found in the study by Nys et al. [6]. Although the prevalence of post-stroke cognitive impairment is high according to previous studies, it appears that the frequency of post-stroke cognitive decline in stroke survivors may have been underestimated $[7,8]$.

The evaluation of cognitive functions by classic neuropsychological methods during an acute stroke is very difficult due to factors that cause the misinterpretation of test results, such as the severity of the patient's condition, apathy, depression, 
and anxiety disorders [9]. So, specific and ideal biomarkers to predict the development of post-stroke cognitive impairment are needed to reflect the severity of its course and effectiveness of treatment [10, 11]. The Alberta Stroke Programme Early Computed Tomography Score (ASPECTS) has been widely used to determine the extent of early ischaemic changes in brain imaging for acute stroke [12].

\section{Clinical rationale for study}

The aim of our study differs from that of other studies in that ASPECTS has been used to predict cognitive impairment resulting from acute ischaemic stroke.

\section{Materials and methods}

\section{Participants and inclusion/exclusion criteria}

This study was approved by the local ethics committee Institutional Research Board (IRB) and all patients provided written consent. 150 patients with acute ischaemic stroke (AIS) were admitted to our neurology department between October 2017 and March 2019; 79 males and 71 females were included in this study and admitted to the Convalescent Care Unit. The 150 had all experienced the first attack of acute MCA territory infarction within two days from the onset and were aged 18 years and older. Acute ischaemic stroke (AIS) was defined as a rapidly developing neurological deficit with an obvious known onset, plus an initial CT brain with no proof of ICH. Exclusion criteria were previous stroke, presence of anterior cerebral artery infarction, posterior cerebral artery infarction, and venous infarction. We also excluded patients with previous neuropsychiatric diseases or who had been prescribed drugs that impair cognition. In addition, severe cases with NIHSS $>25$, and cases where MoCA could not be assessed, were excluded.

\section{Classification and patient subgroups}

Patients were classified into two groups according to their MoCA score three months after the onset of AIS. The first group, the 'cognitively impaired', had a MoCA score of 25 or less. The second group, the 'cognitively preserved', had a MoCA score of 26 or more.

\section{Clinical and radiological diagnosis}

A history of vascular risk factors was obtained for each patient. Complete general and neurological examinations were carried out. All patients underwent $\mathrm{CT}$ examinations by 16-multi-slice GE, (Optima 520, China). Initial non-contrast CT brain was done for all cases at the onset and a follow-up CT brain was done after seven days. For all patients, the CT images were obtained in an axial plane, $5 \mathrm{~mm}$ sections from the base to the vertex. Imaging parameters were: $120 \mathrm{kVp}, 320 \mathrm{~mA}$, FOV of $195 \mathrm{~mm}, 1 \mathrm{~s} /$ rotation, and table speed of $15 \mathrm{~mm} /$ rotation.

Images were analysed independently by neuroradiologists. Assessment of brain CT was done by ASPECTS. An ASPECTS score was calculated for each patient. Brain CT images were assessed for proof of localised parenchymal hypo-attenuation, loss of differentiation between grey and white matter, and if there was effacement of sulci. ASPECTS is a reliable method for the evaluation of ischaemic stroke that utilises a 10-point scoring system, with M1, M2, M3, M4, M5, M6, I: insula, IC: internal capsule, L: lentiform, and C: caudate representing those 10 points. A score of 10 means a normal CT scan. One point is subtracted for each affected area on the brain CT. So, a score of 0 indicates widespread ischaemia affecting the MCA territory (Fig. 1) [13]. Patients were classified into two subgroups, the first group comprising those with better ASPECTS ranging from 10-8, and the second group comprising those with worse ASPECTS ranging from 0-7.

\section{Cognitive assessment}

To assess cognitive functions, we used the Montreal Cognitive Assessment (MoCA) in its Arabic form for our study participants [14]. This scale evaluates different domains of cognition such as attention, orientation, memory, language, visuo-constructional capacity, and executive functions. MoCA is a 30-point test where a score of 25 or below is considered to demonstrate abnormal impaired cognition [15]. Patients were classified into two groups according to their MoCA score three months after the onset of AIS. The first group, the 'cognitively impaired', had a MoCA score of 25 or less. The second group, the 'cognitively preserved' had a MoCA score of 26 or more. Potocnik et al. determined that the optimal cut-off value for impaired cognition is $\leq 25$ points, with high sensitivity ( $81 \%)$ and specificity (70\%) [16].

\section{Statistical analysis}

Quantitative data was prescribed as interquartile and median range and comparison was done by Mann-Whitney $\mathrm{U}$ test. Nominal data was presented as numbers and percentages and compared by the $\chi^{2}$ test.

In order to reveal possible correlations between cognitive impairment and ALBERTA, the Pearson correlation analysis was used. Multivariate logistic regression analysis was carried out for the independent variables of cognitive impairment such as age, ASPECTS, and MoCA domains.

\section{Results}

Thirty-eight $(25.3 \%)$ ischaemic stroke patients showed impaired cognitive functions, while 112 patients $(74.7 \%)$ had preserved cognition. Patients with impaired cognitive functions were older $(p=0.001)$ with no significant sex difference $(\mathrm{p}=0.71)($ Tab. 1$)$.

Patients with impaired cognitive functions compared to patients with preserved cognition were associated with a significantly higher incidence of hypertension $(\mathrm{p}=0.001)$, but there was no significant difference between the two groups regarding DM, smoking, IHD, AF, or hyperlipidemia. 

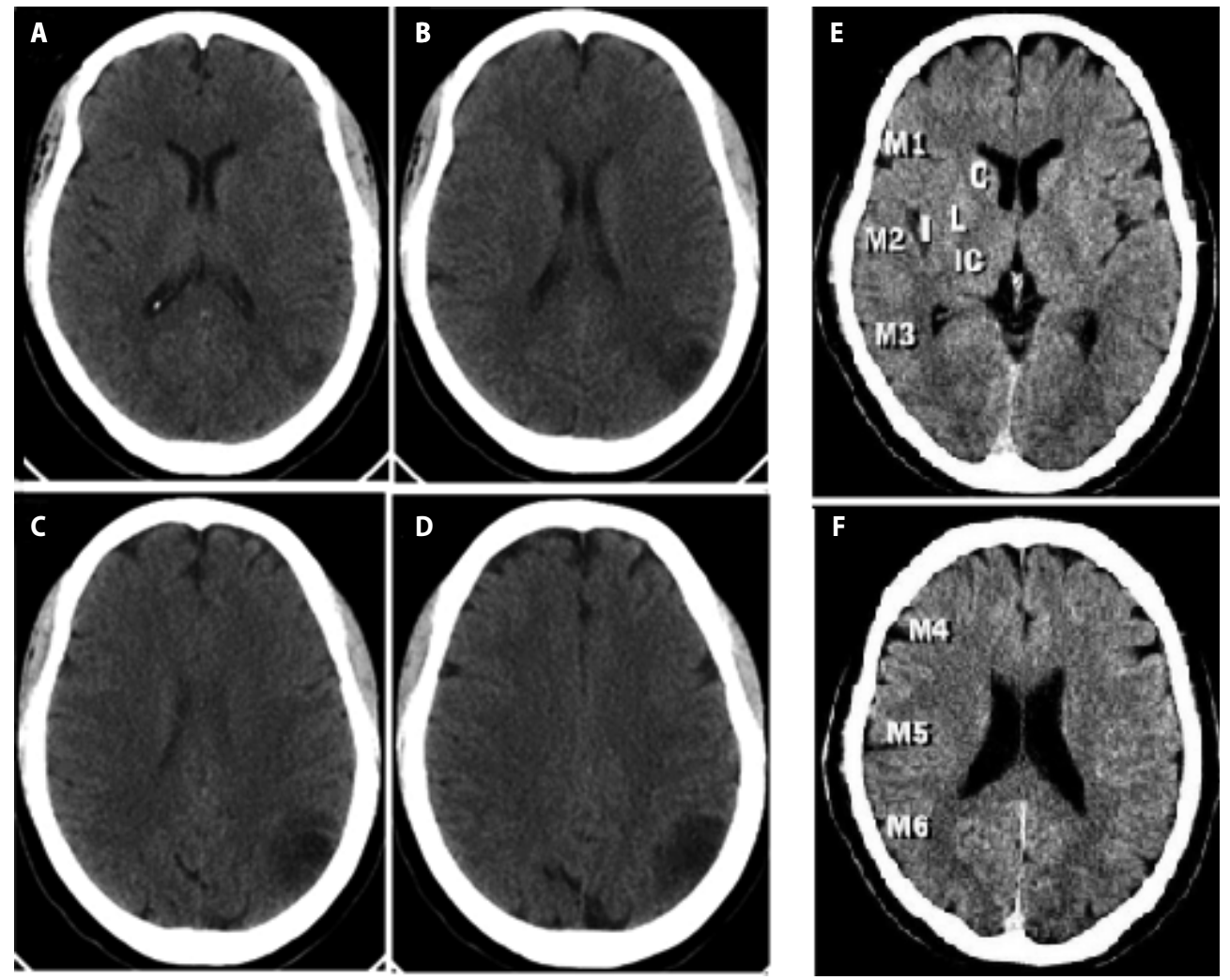

Figure 1. A-D sequential axial non-contrast CT images of brain reveal hypodense area of infarction at left posterior parietal region (M3 and M6 regions); ASPECTS $=8$. E-F sequential axial non-contrast CT images of brain reveal ganglionic and supraganglionic levels and ASPECTS scoring: one point is subtracted for each affected area on brain CT

In addition, there was a significantly higher initial NIHSS in patients with impaired cognitive functions compared to patients with preserved cognition $(19.32 \pm 5.74$ vs. 12.34 \pm 6.69 and $p=0.001)$. Moreover, cognitively impaired patients were associated with more severe and moderate NIHSS scores compared to cognitively preserved patients $(\mathrm{p}=0.002)($ Tab. 1$)$.

There was a clear significant impairment in the domains of language, attention, memory, and executive functions $(\mathrm{p}<0.005)$. On the other hand, the domains of visual-spatial ability, naming, and orientation were slightly decreased in cognitively impaired patients (Tab. 1).

Also, there was a strong positive correlation between ASPECTS and total MoCA test $(r=0.73$ and $p=0.002)$, as shown in Figure 2.

Regarding hemispheric dominance, cases with dominant hemisphere infarction demonstrated significantly lower MoCA scores compared to cases with non-dominant hemispheric infarction ( $22.36 \pm 5.42$ vs. $23 \pm 3.92, \mathrm{p}=0.029)$, as demonstrated in Figure 3.

Finally, logistic regression analysis demonstrated the independent factors associated with cognitive impairment to be older age (OR 2.42, $\mathrm{p}<0.001$ ), followed by certain domains of the MoCA test like executive functions, memory, attention, and language (OR 2.11, p = 0.001, OR 2.1, p = 0.001, OR 2.06, $\mathrm{p}=0.004$, and OR 2.09, $\mathrm{p}=0.002$ respectively), and NIHSS and
HTN (OR 2.1, p $=0.001$ and OR 2.03, p $=0.006$ respectively). Also, although to a lesser extent, ASPECTS was associated with cognitive impairment (OR 2.01, $\mathrm{p}=0.005)(\mathrm{Tab} .2)$.

\section{Discussion}

Our study had two goals: (1) to identify the prevalence of cognitive impairment in AIS, and (2) to assess if there is a correlation between ASPECTS and cognitive outcomes after three months.

Post-stroke impaired cognition occurs frequently, its prevalence ranging from $20-80 \%$ with differences between countries, races, and diagnostic criteria. The risk of post-stroke impaired cognition is related to both demographic factors like age, education, and occupation, and vascular factors [17].

Acute ischaemic stroke (AIS) requires a rapid evaluation of clinical and radiological findings. The ability to distinguish an acute infarct by $\mathrm{CT}$ is helpful in confirming the diagnosis and the analysis of acute stroke [18]. CT has the advantage of being a simple technique that leaves enough time for early treatment and fast intervention where needed. Baseline ASPECTS is a reliable predictor of prognosis in patients with AIS [19]. ASPECTS is increasingly being integrated into the decision-making process for intervention in patients with AIS [20]. 
Table 1. Patients with cognitive impairment compared to patients with preserved cognition

\begin{tabular}{|c|c|c|c|}
\hline & Impaired cognition & Preserved cognition & P value \\
\hline Number & $38(25.3 \%)$ & $112(74.7 \%)$ & \\
\hline Age $(y)$ mean $\pm S D$ & $68.5 \pm 7.12$ & $60.23 \pm 7.61$ & $0.001^{*}$ \\
\hline Age $<60 \mathrm{~N}(\%)$ & 13 & 71 & $0.002^{*}$ \\
\hline Age $>60 \mathrm{~N}(\%)$ & 25 & 41 & \\
\hline Gender (male) & $21(55.2 \%)$ & $58(51.8 \%)$ & 0.71 \\
\hline Hypertension & $30(79.8 \%)$ & $56(50 \%)$ & $0.001^{*}$ \\
\hline DM & $11(29 \%)$ & $28(25 \%)$ & 0.63 \\
\hline Smoking & $15(39.5 \%)$ & 37 (33\%) & 0.47 \\
\hline Hyperlipidemia & $7(18.4 \%)$ & $16(14.3 \%)$ & 0.54 \\
\hline $\mathrm{AF}$ & $7(18.4 \%)$ & $18(16 \%)$ & 0.73 \\
\hline IHD & $4(10.5 \%)$ & $11(9.2 \%)$ & 0.9 \\
\hline Initial NIHSS mean \pm SD & $19.32 \pm 5.74$ & $12.34 \pm 6.69$ & 0.001 \\
\hline Mild (0-5) & 9 & 61 & 0.002 \\
\hline Moderate (6-15) & 13 & 29 & \\
\hline Severe $(\geq 16)$ & 16 & 22 & \\
\hline \multicolumn{4}{|l|}{ MoCA test scores } \\
\hline Visuo-spatial ability & $3.18 \pm 1.23$ & $3.4 \pm 0.89$ & 0.39 \\
\hline Naming & $2.25 \pm 0.91$ & $2.58 \pm 0.72$ & 0.21 \\
\hline Executive functions & $2.28 \pm 1.29$ & $3.7 \pm 0.95$ & $p<0.001$ \\
\hline Attention & $4.51 \pm 1.28$ & $5.93 \pm 0.96$ & 0.004 \\
\hline Language & $3.41 \pm 1.02$ & $4.42 \pm 1.12$ & 0.002 \\
\hline Memory & $2.82 \pm 1.41$ & $4.19 \pm 1.11$ & $p<0.001$ \\
\hline Orientation & $5.82 \pm 0.39$ & $6.49 \pm 0.34$ & 0.11 \\
\hline Total MoCA score & $21.72 \pm 2.93$ & $26.02 \pm 3.21$ & $p<0.001$ \\
\hline ASPECTS & $5.23 \pm 3.95$ & $8.54 \pm 1.44$ & $p=0.005$ \\
\hline
\end{tabular}

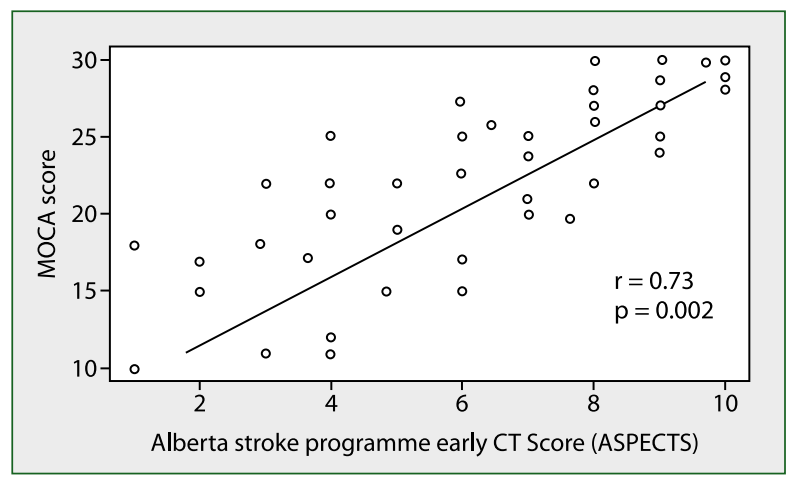

Figure 2. Correlation of ASPECTS against MoCA score

Prevalence of post-stroke cognitive impairment

The prevalence of post-stroke $\mathrm{CI}$ in previous studies ranged from $7.5-72 \%$ [21-24]. In the present study, the prevalence of cognitive impairment was $25.3 \%$, similar to the percentage found in previous studies. The varying prevalence in previous studies might be due to differences in the setting of a study,

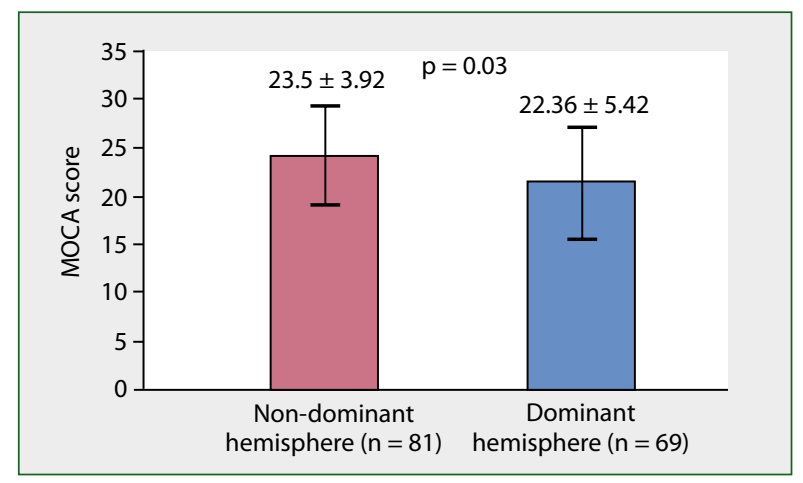

Figure 3. Relationship of laterality of infarction and cognition

different age groups, and different assessment scales and biomarkers used to diagnose cognitive impairment.

For example, a study by Liman et al. reported $11.8 \%$ cognitive impairment three years after stroke [25]. Douiri et al. reported $24 \%$ cognitive impairment three months from onset [26], and Knopman et al. reported $10.9 \%$ cognitive impairment 
Table 2. Independent factors highly associated with cognitive impairment

\begin{tabular}{lccc}
\multicolumn{1}{c}{ Variables } & O R & $95 \% \mathbf{C l}$ & $\mathbf{P}$ \\
Age & 2.42 & $1.87-3.12$ & $\mathrm{p}<0.001$ \\
HTN & 2.03 & $1.37-2.67$ & 0.005 \\
NIHSS & 2.1 & $1.51-2.73$ & 0.001 \\
ASPECTS & 2.01 & $1.39-2.64$ & $\mathrm{p}=0.005$ \\
Executive functions & 2.11 & $1.53-2.79$ & $\mathrm{p}=0.001$ \\
Attention & 2.06 & $1.43-2.68$ & $\mathrm{p}=0.004$ \\
Language & 2.09 & $1.46-2.71$ & $\mathrm{p}=0.002$ \\
Memory & 2.1 & $1.45-2.75$ & $\mathrm{p}=0.001$
\end{tabular}

[27]. The difference in the prevalence of impaired cognition in our study compared to the other studies was due to the scale used which was MoCA; previous studies used MMSE with a cut-off score of $<24$ to indicate impaired cognition. Although MMSE is sensitive in diagnosing dementia, it is insensitive in diagnosing early dementia or mild cognitive impairment [28, 29].

\section{Risk factors of AIS and cognitive impairment}

In our study, the common risk factors for stroke were hypertension, smoking, DM, AF, hyperlipidemia, and ischaemic heart disease. These findings are more or less similar to the studies by Amelia et al. and Mahdi et al. [30, 31]. Moreover, recent studies agree with our results regarding the traditional risk factors such as hypertension, diabetes, dyslipidemia, and smoking that can account for $60-80 \%$ of strokes [32-34].

Patients with impaired cognitive functions compared to patients with preserved cognition were older $(\mathrm{p}=0.001)$ and showed a significantly higher incidence of hypertension $(\mathrm{p}=0.001)$, but there was no significant difference between the two groups regarding sex, DM, smoking, IHD, AF, or hyperlipidemia. Also, the study by Goldstein et al. on stroke survivors with high blood pressure found impaired cognitive function, especially in the performance of tasks requiring rapid responses and expressive language [35]. The literature has explained the role of vascular risk factors and the mechanism of impaired cognition [36, 37], and the interaction of vascular risk factors with ischaemic stroke [38].

Infections are known to increase the risk of stroke. Also, infection in stroke patients is a common inpatient complication. On top of the fact that infection can lead to cerebral stroke, stroke also induces suppression of immunity which increases the chance of infection and adversely influences cognitive outcomes in patients with stroke, all serving to increase the risk of cognitive impairment following stroke $[39,40]$.

\section{Correlations of ASPECTS, NIHSS, and laterality of infarction with cognition}

We found a significant positive correlation between the neurocognitive test domains (executive functions, attention, memory, and language) and ASPECTS ( $\mathrm{p}<0.05)$. Cognitively impaired patients were associated with more severe and moderate NIHSS scores compared to cognitively preserved patients. Similarly, analysing the results of Sivakumar et al. by Spearman correlation indicated that NIHSS scores were significantly correlated with MoCA scores at baseline $(\mathrm{r}=-0.52 ; \mathrm{p}=0.02)$ and at day $30(\mathrm{r}=-0.51 ; \mathrm{p}=0.04)[41]$. We also detected that cases of ischaemic stroke with dominant hemispheric infarction showed lower scores on the MoCA test. These results were similar to the findings of Chan et al., who found that poor performance of dominant cerebral hemisphere ischaemic stroke cases on the MoCA test probably indicates the reliance of the MoCA test sub-items on both receptive and expressive language abilities beside verbal memory capacities, which commonly affect the dominant cerebral hemisphere in stroke [42].

\section{Factors associated with cognitive impairment in ischaemic stroke}

Finally, logistic regression analysis showed that the independent factors associated with impaired cognition in ischaemic stroke were older age, hypertension, NIHSS, and certain domains of the MoCA test like executive functions, memory, attention, and language $(\mathrm{p}<0.005)$. To a lesser extent, ASPECTS was associated with cognitive impairment $(\mathrm{p}<0.01)$.

The management of impaired cognition following stroke will include anti-dementia medications and therapeutic measures for cerebrovascular diseases. As impaired cognition following stroke is attributed to cerebral lesions caused by ischaemic injury, it seems likely that an improved ischaemic injury could ameliorate cognitive improvement. There is increasing evidence that managing vascular risk factors related to stroke could decrease the risk of post-stroke cognitive impairment [43].

A combination of antiplatelet treatment and clopidogrel therapy should be considered in cases with cerebral ischaemic stroke for the prevention of recurrent stroke with subsequent cognitive impairment [44].

\section{Clinical implications/future directions}

We conclude that the prevalence of cognitive impairment is about $25 \%$ in acute ischaemic stroke patients after three months of follow-up. ASPECTS is directly correlated with cognitive impairment via a positive correlation with the results of neuropsychological testing (MoCA), and may be useful in anticipating post-stroke cognitive impairment. Also, early diagnosis or the anticipation of future impaired cognition following ischaemic stroke will be of therapeutic importance. Both the long-term follow-up of patients and the assessment of their cognitive functions, together with the medications that improve cognition, are advised.

Funding: This publication was prepared without any external source of funding.

Conflicts of interest: None.

Acknowledgements: This research received no specific grant from any funding agency. 


\section{References}

1. GBD 2016 Neurology Collaborators, GBD 2016 Stroke Collaborators. Global, regional, and national burden of stroke, 1990-2016: a systematic analysis for the Global Burden of Disease Study 2016. Lancet Neurol. 2019; 18(5): 439-458, doi: 10.1016/S1474 4422(19)30034-1, indexed in Pubmed: 30871944.

2. Kalaria RN, Akinyemi R, Ihara M. Stroke injury, cognitive impairment and vascular dementia. Biochim Biophys Acta. 2016; 1862(5): 915-925, doi: 10.1016/j.bbadis.2016.01.015, indexed in Pubmed: 26806700.

3. Jacova C, Pearce LA, Roldan AM, et al. Cognitive impairment in lacunar strokes: the SPS3 trial. Ann Neurol. 2012; 72(3): 351-362, doi: 10.1002/ana.23733, indexed in Pubmed: 23034910.

4. Nakling AE, Aarsland D, Næss H, et al. Cognitive Deficits in Chronic Stroke Patients: Neuropsychological Assessment, Depression, and Self-Reports. Dement Geriatr Cogn Dis Extra. 2017; 7(2): 283-296, doi: 10.1159/000478851, indexed in Pubmed: 29033974.

5. Schaapsmeerders P, Tuladhar AM, Arntz RM, et al. Long-term cognitive impairment after first-ever ischemic stroke in young adults. Stroke. 2013; 44(6): 1621-1628, doi: 10.1161/STROKEAHA.111.000792, indexed in Pubmed: 23652272.

6. Nys GMS, van Zandvoort MJE, de Kort PLM, et al. Restrictions of the Mini-Mental State Examination in acute stroke. Arch Clin Neuropsychol. 2005; 20(5): 623-629, doi: 10.1016/j.acn.2005.04.001, indexed in Pubmed: 15939186.

7. Pendlebury ST, Cuthbertson FC, Welch SJV, et al. Underestimation of cognitive impairment by Mini-Mental State Examination versus the Montreal Cognitive Assessment in patients with transient ischemic attack and stroke: a population-based study. Stroke. 2010; 41(6): 1290-1293, doi: 10.1161/STROKEAHA.110.579888, indexed in Pubmed: 20378863.

8. Bour A, Rasquin S, Boreas A, et al. How predictive is the MMSE for cognitive performance after stroke? J Neurol. 2010; 257(4): 630-637, doi: 10.1007/s00415-009-5387-9, indexed in Pubmed: 20361295.

9. Fanning JP, Wong AA, Fraser JF. The epidemiology of silent brain infarction: a systematic review of population-based cohorts. BMC Med. 2014; 12: 119, doi: 10.1186/s12916-014-0119-0, indexed in Pubmed: 25012298.

10. Hsieh YC, Seshadri S, Chung WT, et al. Formosa Stroke Genetic Consortium (FSGC). Association between genetic variant on chromosome 12p13 and stroke survival and recurrence: a one year prospective study in Taiwan. J Biomed Sci. 2012; 19: 1, doi: 10.1186/1423-012719-1, indexed in Pubmed: 22212150.

11. Riverol M, López OL. Biomarkers in Alzheimer's disease. Front Neurol. 2011; 2: 46, doi: 10.3389/fneur.2011.00046, indexed in Pubmed: 21808632.

12. Schröder J, Thomalla G. A Critical Review of Alberta Stroke Program Early CT Score for Evaluation of Acute Stroke Imaging. Front Neurol. 2016; 7: 245, doi: 10.3389/fneur.2016.00245, indexed in Pubmed: 28127292

13. Pexman JH, Barber PA, Hill MD, et al. Use of the Alberta Stroke Program Early CT Score (ASPECTS) for assessing CT scans in patients with acute stroke. AJNR Am J Neuroradiol. 2001; 22(8): 1534-1542, indexed in Pubmed: 11559501.

14. Nasreddine ZS, Phillips NA, Bédirian V, et al. The Montreal Cognitive Assessment, MoCA: a brief screening tool for mild cognitive impairment. J Am Geriatr Soc. 2005; 53(4): 695-699, doi: 10.1111/j.1532-5415.2005.53221.x, indexed in Pubmed: 15817019.
15. Tan Jp, Li N, Gao J, et al. Optimal cutoff scores for dementia and mild cognitive impairment of the Montreal Cognitive Assessment among elderly and oldest-old Chinese population. J Alzheimers Dis. 2015; 43(4): 1403-1412, doi: 10.3233/JAD-141278, indexed in Pubmed: 25147113.

16. Potocnik J, Ovcar Stante K, Rakusa M. The validity of the Montreal cognitive assessment (MoCA) for the screening of vascular cognitive impairment after ischemic stroke. Acta Neurol Belg. 2020; 120(3): 681-685, doi: 10.1007/s13760-020-01330-5, indexed in Pubmed: 32193731.

17. Sun JH, Tan L, Yu JT. Post-stroke cognitive impairment: epidemiology, mechanisms and management. Ann Transl Med. 2014; 2(8): 80, doi: 10.3978/j.issn.2305-5839.2014.08.05, indexed in Pubmed: 25333055.

18. Etherton MR, Barreto AD, Schwamm LH, et al. Neuroimaging Paradigms to Identify Patients for Reperfusion Therapy in Stroke of Unknown Onset. Front Neurol. 2018; 9: 327, doi: 10.3389/ fneur.2018.00327, indexed in Pubmed: 29867736.

19. Ryu CW, Shin HS, Park S, et al. Alberta Stroke Program Early CT Score in the Prognostication after Endovascular Treatment for Ischemic Stroke: A Meta-analysis. Neurointervention. 2017; 12(1): 20-30, doi: 10.5469/neuroint.2017.12.1.20, indexed in Pubmed: 28316866.

20. Sundaram VK, Goldstein J, Wheelwright D, et al. Automated ASPECTS in Acute Ischemic Stroke: A Comparative Analysis with CT Perfusion. AJNR Am J Neuroradiol. 2019; 40(12): 2033-2038, doi: 10.3174/ ajnr.A6303, indexed in Pubmed: 31727750.

21. Sachdev PS, Brodaty $H$, Valenzuela MJ, et al. Clinical determinants of dementia and mild cognitive impairment following ischaemic stroke: the Sydney Stroke Study. Dement Geriatr Cogn Disord. 2006; 21(5-6): 275-283, doi: 10.1159/000091434, indexed in Pubmed: 16484805

22. Claesson L, Lindén T, Skoog I, et al. Cognitive impairment after stroke - impact on activities of daily living and costs of care for elderly people. The Göteborg 70+ Stroke Study. Cerebrovasc Dis. 2005; 19(2): $102-$ 109, doi: 10.1159/000082787, indexed in Pubmed: 15608434.

23. Cao M, Ferrari M, Patella R, et al. Neuropsychological findings in young-adult stroke patients. Arch Clin Neuropsychol. 2007; 22(2): 133-142, doi: 10.1016/j.acn.2006.09.005, indexed in Pubmed: 17169527.

24. Cengić $L$, Vuletić $V$, Karlić $M$, et al. Motor and cognitive impairment after stroke. Acta Clin Croat. 2011; 50(4): 463-467, indexed in Pubmed: 22649874.

25. Liman TG, Heuschmann PU, Endres M, et al. Changes in cognitive function over 3 years after first-ever stroke and predictors of cognitive impairment and long-term cognitive stability: the Erlangen Stroke Project. Dement Geriatr Cogn Disord. 2011; 31(4): 291-299, doi: 10.1159/000327358, indexed in Pubmed: 21502760.

26. Douiri A, Rudd AG, Wolfe CDA. Prevalence of poststroke cognitive impairment: South London Stroke Register 1995-2010. Stroke. 2013; 44(1): 138-145, doi: 10.1161/STROKEAHA.112.670844, indexed in Pubmed: 23150656.

27. Knopman DS, Roberts RO, Geda YE, et al. Association of prior stroke with cognitive function and cognitive impairment: a population-based study. Arch Neurol. 2009; 66(5): 614-619, doi: 10.1001/archneurol.2009.30, indexed in Pubmed: 19433661.

28. Bour A, Rasquin S, Boreas A, et al. How predictive is the MMSE for cognitive performance after stroke? J Neurol. 2010; 257(4): 630-637, doi: 10.1007/s00415-009-5387-9, indexed in Pubmed: 20361295. 
29. Sundar U, Adwani S. Post-stroke cognitive impairment at 3 months. Ann Indian Acad Neurol. 2010; 13(1): 42-46, doi: 10.4103/09722327.61276, indexed in Pubmed: 20436746.

30. Boehme AK, Esenwa C, Elkind MSV. Stroke Risk Factors, Genetics, and Prevention. Circ Res. 2017; 120(3): 472-495, doi: 10.1161/CIRCRESAHA.116.308398, indexed in Pubmed: 28154098.

31. Habibi-Koolaee M, Shahmoradi L, Niakan Kalhori SR, et al. Prevalence of Stroke Risk Factors and Their Distribution Based on Stroke Subtypes in Gorgan: A Retrospective Hospital-Based Study-2015-2016. Neurol Res Int. 2018; 2018: 2709654, doi: 10.1155/2018/2709654, indexed in Pubmed: 30147952.

32. Kim JS. Unconventional, Yet Important, Risk Factors for Stroke. J Stroke. 2018; 20(1): 1, doi: 10.5853/jos.2018.00241, indexed in Pubmed: 29402061.

33. Bang OhY, Ovbiagele B, Kim JS. Nontraditional Risk Factors for Ischemic Stroke: An Update. Stroke. 2015; 46(12): 3571-3578, doi: 10.1161/STROKEAHA.115.010954, indexed in Pubmed: 26514189.

34. Kim YD, Jung YoH, Saposnik G. Traditional Risk Factors for Stroke in East Asia. J Stroke. 2016; 18(3): 273-285, doi: 10.5853/ jos.2016.00885, indexed in Pubmed: 27733028.

35. Goldstein FC, Levey Al, Steenland NK. High blood pressure and cognitive decline in mild cognitive impairment. J Am Geriatr Soc. 2013; 61(1): 67-73, doi: 10.1111/jgs.12067, indexed in Pubmed: 23301925.

36. Sun JH, Tan L, Yu JT. Post-stroke cognitive impairment: epidemiology, mechanisms and management. Ann Transl Med. 2014; 2(8): 80, doi: 10.3978/j.issn.2305-5839.2014.08.05, indexed in Pubmed: 25333055.

37. Swartz RH, Bayley M, Lanctôt KL, et al. Post-stroke depression, obstructive sleep apnea, and cognitive impairment: Rationale for, and barriers to, routine screening. Int J Stroke. 2016; 11(5): 509-518, doi: 10.1177/1747493016641968, indexed in Pubmed: 27073189.
38. Ovbiagele B, Saver JL, Sanossian N, et al. Predictors of cerebral microbleeds in acute ischemic stroke and TIA patients. Cerebrovasc Dis. 2006; 22(5-6): 378-383, doi: 10.1159/000094855, indexed in Pubmed: 16888379.

39. Elkind MSV, Boehme AK, Smith CJ, et al. Infection as a Stroke Risk Factor and Determinant of Outcome After Stroke. Stroke. 2020; 51(10): 3156-3168, doi: 10.1161/STROKEAHA.120.030429, indexed in Pubmed: 32897811.

40. Kocatürk M, Kocatürk Ö. Assessment of relationship between C-reactive protein to albumin ratio and 90-day mortality in patients with acute ischaemic stroke. Neurol Neurochir Pol. 2019; 53(3): 205-211, doi: 10.5603/PJNNS.a2019.0020, indexed in Pubmed: 31145464.

41. Sivakumar L, Kate M, Jeerakathil T, et al. Serial montreal cognitive assessments demonstrate reversible cognitive impairment in patients with acute transient ischemic attack and minor stroke. Stroke. 2014; 45(6): 1709-1715, doi: 10.1161/STROKEAHA.114.004726, indexed in Pubmed: 24757105.

42. Chan $\mathrm{E}$, Altendorff $\mathrm{S}$, Healy $\mathrm{C}$, et al. The test accuracy of the Montreal Cognitive Assessment (MoCA) by stroke lateralisation. J Neurol Sci. 2017; 373: 100-104, doi: 10.1016/j.jns.2016.12.028, indexed in Pubmed: 28131163.

43. Sun JH, Tan L, Yu JT. Post-stroke cognitive impairment: epidemiology, mechanisms and management. Ann Transl Med. 2014; 2(8): 80, doi: 10.3978/j.issn.2305-5839.2014.08.05, indexed in Pubmed: 25333055.

44. Wiśniewski A, Sikora J, Filipska K, et al. Assessment of the relationship between platelet reactivity, vascular risk factors and gender in cerebral ischaemia patients. Neurol Neurochir Pol. 2019; 53(4): 258-264, doi: 10.5603/PJNNS.a2019.0028, indexed in Pubmed: 31343071. 